\title{
Research Paper \\ The Relationship Between Memory Impairment and Health Indicators of the Elderly With Parkinson Disease
}

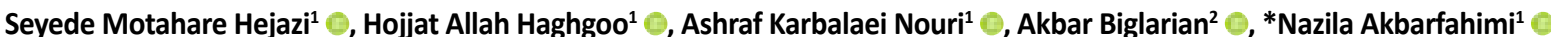

1. Department of Occupational Therapy, University of Social Welfare and Rehabilitation Sciences, Tehran, Iran

2. Department of Biostatistics, Social Determinants of Health Research Center, University of Social Welfare and Rehabilitation Sciences, Tehran, Iran.

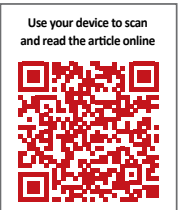

Cftation Hejazi SM, Haghgoo H, Karbalaei Nouri A, Biglarian A, Akbarfahimi N. [The Relationship Between Memory Impairment and Health Indicators of the Elderly With Parkinson Disease (Persian)]. Salmand: Iranian Journal of Ageing. 2019; 13(Special Issue):666-675. https://doi.org/10.32598/SIJA.13.Special Issue.666

d. https://doi.org/10.32598/SIJA.13.Special-Issue.666

Key words: Parkinson disease, Aging, Memory impairment, Health indicators

\section{A B S TRACT}

Objectives Parkinson Disease (PD) is a progressive neurodegenerative disorder affecting motor and cognitive functions. Cognitive impairments are related to different causes such as health indicators. This study aimed to examine the relationship between cognitive impairment and health indicators of patients with PD.

Methods \& Materials This study was a cross-sectional and descriptive study. By convenience sampling method, a total of 30 PD patients 60-70 years old, diagnosed by a neurologist, were recruited. Cognitive Montreal Test was administrated to measure their global cognitive function. Rey visual test was used to determine their visual memory and the Wechsler test (adult memory) was used to assess their verbal memory. Their sleep quality was assessed by the Pittsburgh Sleep Quality Index. Demographic information and health indicators such as blood pressure were collected via interview. The obtained data were analyzed in SPSS 22.

Results The results showed significant relationship between some indicators such as blood pressure with verbal memory $(r=-0.514, P=0.004)$, and sleep disorder $(r=-0.421, P=0.031)$, and visual memory $(r=0.368$, $\mathrm{P}=0.045)$.

Conclusion This research revealed that blood pressure and sleep disturbances can affect memory function. Therefore, the cognitive problems in people with PD can be postponed by screening these factors and controlling them with early medical interventions.

\section{Extended Abstract}

\section{Objectives}

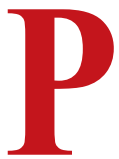

arkinson Disease (PD) is a progressive neurological disease that causes motor dysfunction and cognitive decline and consequently creates many problems for the affected pa- tients and their caregivers [1-3]. The prevalence of PD increases dramatically after age 60 [2]. Aging is one of the biggest risk factors for the development of $\mathrm{PD}$, such that its prevalence in people aged $45-55$ years is $0.02 \%$ and is estimated to reach $85.77 \%$ by the age of 85 [4]. On the other hand, some disorders and illnesses such as sleep disorder and impaired blood pressure can make a person more prone to cognitive impairments [5-8]. Regarding the effect of

* Corresponding Author:

Nazila Akbar Fahimi, PhD.

Address: Department of Occupational Therapy, University of Social Welfare and Rehabilitation Sciences, Tehran, Iran.

Tel: +98 (21) 22180063

E-mail: fahimi1970@yahoo.com 
cognitive impairment on several factors, including health indicators, this study attempted to evaluate the relationship between visual and verbal memory impairment with health indicators of the elderly with PD.

\section{Methods \& Materials}

This cross-sectional study was conducted on 30 older adults with PD living in Tehran (diagnosed by a neurologist) in 2017. They were selected based on convenience sampling method and the inclusion criteria were being 6070 years old, having PD for 1-6 years, being able to read and write, being able to establish verbal communication and follow instructions, and lacking aphasia and linguistic problems. The exclusion criteria were the unwillingness to participate and returning incomplete questionnaires. After obtaining written informed consent from the participants, the data collection process was started. At first, the Montreal cognitive assessment tool was used to screen for cognitive impairment.

Because of the high prevalence of visual and verbal memory impairment among PD patients, Wechsler memory scale was used to determine the verbal memory impairment, and Rey 15-item visual memory test was used to check the visual memory impairment. Moreover, to measure sleep quality of the subjects, the Pittsburgh sleep quality index was employed. The existence of blood pressure disorder in subjects was determined based on their medical records and specialist's diagnosis. After collecting the study data, they were analyzed in SPSS V. 22.

\section{Results}

Of 30 participants, 15 were male and 15 females with a Mean \pm SD age of $64.76 \pm 7.87$ years. More than $50 \%$ had high blood pressure and over $60 \%$ had sleep disorders. More than $96 \%$ of them had a visual impairment and $70 \%$ had verbal memory. Based on the results of the Shapiro-Wilk test, all study variables had a normal distribution $(\mathrm{P}>0.05)$.
Based on a study, the prevalence of high blood pressure in Iranian people (Birjand City dwellers) was reported 20\% [9]. In this study, it was more than $50 \%$. This indicates a higher incidence of high blood pressure in the elderly with PD compared to the normal elderly population. In this regard, and considering the nature of PD in causing a disturbance between the acetylcholine and dopaminergic systems which leads to the dysfunction of acetylcholine system, imbalance of sympathetic nervous system and blood pressure [10], it can be said that high blood pressure can be a factor in exacerbating cognitive deficits.

According to the participants' reports and based on PSQI results, symptoms such as tremor during sleep, activation of sympathetic nervous system, frequent urination, and sleep disturbances were the main causes of poor sleep quality which can affect the function of the brain and the information relay between the hippocampus and the cortex, and subsequently cognitive defects [11].

In order to examine the relationship between visual and verbal memory impairment with blood pressure and sleep disorders, the Pearson correlation test was used whose results are shown in Table 1 . Based on the results, visual $(\mathrm{P}=0.031)$ and verbal $(\mathrm{P}=0.004)$ memory impairment had a significant correlation with high blood pressure $(\mathrm{P}<0.005)$. Between visual memory impairment and sleep disorder, there was also a significant correlation $(\mathrm{P}=0.045)$, but verbal memory impairment had no significant association with sleep disorder $(\mathrm{P}>0.05)$.

\section{Conclusion}

There is a significant relationship between memory impairment and health indicators of older people with PD. Therefore, it can be expected that by examining these factors and controlling them with timely medical interventions, the incidence and severity of cognitive impairment in these individuals can be manipulated and decreased. In order to better assess the memory status of the elderly people with $\mathrm{PD}$, it is suggested that their narrative memory impairment with declarative memory impairment be compared using a larger sample size. Regarding the relationship

Table 1. The Pearson correlation coefficient between visual and verbal memory impairment and health indicators

\begin{tabular}{ccccc}
\hline \multirow{2}{*}{ Variable } & \multicolumn{2}{c}{ High Blood Pressure } & \multicolumn{2}{c}{ Sleep Disorder } \\
\cline { 2 - 5 } & $\mathbf{r}$ & .Sig & $\mathbf{r}$ & .Sig \\
\hline Verbal memory impairment & -0.514 & 0.004 & 0.307 & 0.099 \\
Visual memory impairment & -0.421 & 0.031 & 0.368 & 0.045 \\
\hline
\end{tabular}


between sleep disorder and memory impairment in people with PD, the sleep quality improvement techniques should be taught to them by proper rehabilitation and using physical interventions. Moreover, it is recommended that serious professional rehabilitation principles be reconsidered in occupational and social activities after 2 years.

\section{Ethical Considerations}

Compliance with ethical guidelines

This paper has been approved by University of Social Welfare and Rehabilitation sciences IR.USWR.REC.1396.105. The research goals and process were explained to the participants and they were assured of the confidentiality of their information. They were free to quit the study at any time.

Funding

This paper was extracted from the MSc. thesis of Motahare Hejazi in Department of Occupational Therapy, University of Social Welfare and Rehabilitation Sciences. This research received specific grant from Clinical Research Development Center of Rofeideh Rehabilitation Hospital.

\section{Authors' contributions}

Conceptualization: Seyede Motahare Hejazi, Nazila Akbarfahimi, Hojjat Allah Haghgoo, Ashraf Karbalaei Nouri; Methodology: Seyede Motahare Hejazi; Foramal analysis: Akbar Biglarian, Nazila Akbarfahimi; Writing review: Nazila Akbarfahimi, Hojjat Allah Haghgoo, Seyede Motahare Hejazi; and Final Editing: Nazila Akbarfahimi, Hojjat Allah Haghgoo.

\section{Conflict of interest}

The authors declared no conflict of interest.

\section{Acknowledgments}

Authors are grateful to all the elderly people participating in this study, their care givers, the Chancellor and the staff of the Rofeideh rehabilitation hospital who helped authors to do this study. 


\title{
ارتباط بين اختلال حافظه (بينايى و كلامى) با شاخصهاى سلامت سالمندان داراى ياركينسون
}

\author{
سيده مطهره حجازى' •، حجتاله حقكو' • اشرف كربلايى نورى' • اكبر بيكلريان' • •نازيلا اكبرفهيمى' \\ 1- كروه كاردرمانى، دانشكاه علوم بهزيستى و توانبخشى، تهرانه ايران.

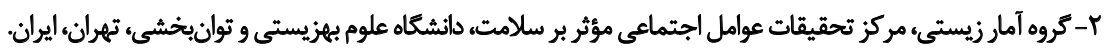

\begin{abstract}
حكSد

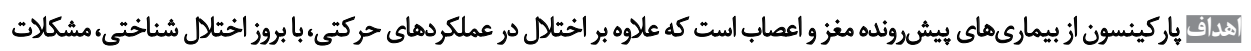

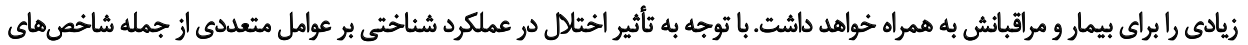

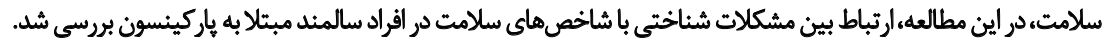

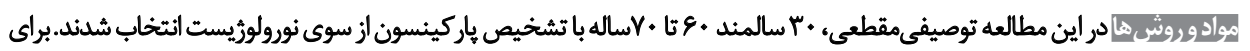

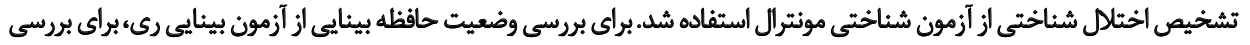

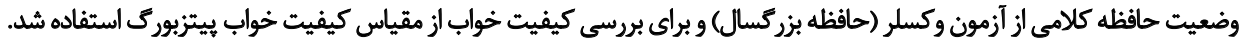

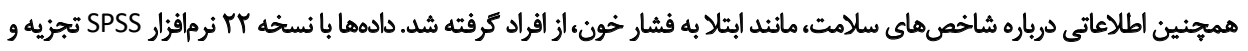
تحليل شدند.

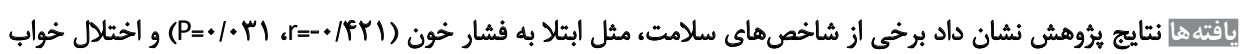

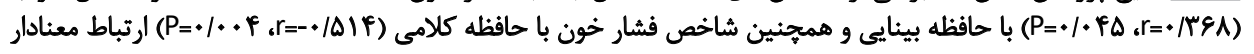

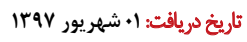

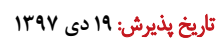

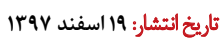

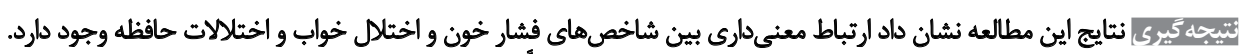

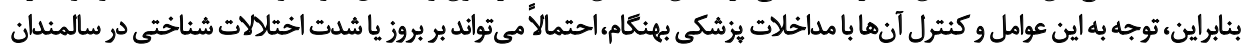
مبئلا به باركينسون مؤثر باشد.

كليدوارٔهاها:

باركينسونء سالمند، اختلال حافظةه. شاخص هاى سلامت حافظه

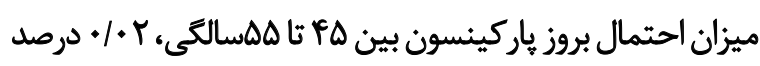

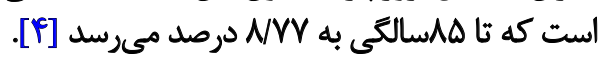

مقدمه

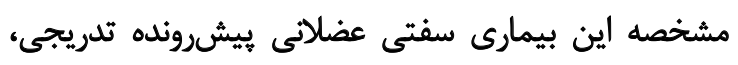

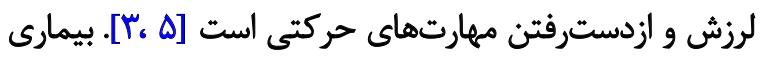

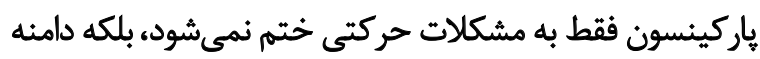

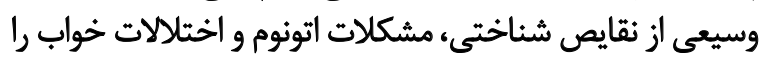

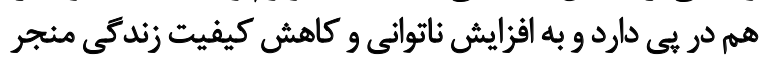

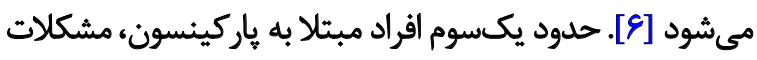

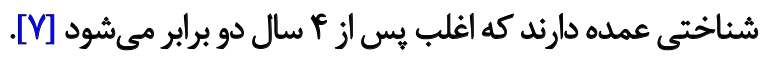

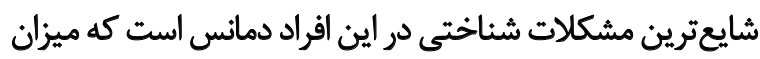

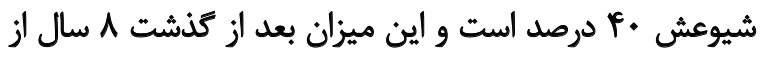

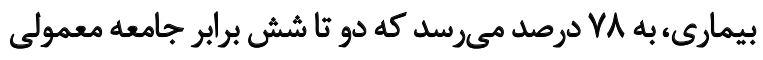

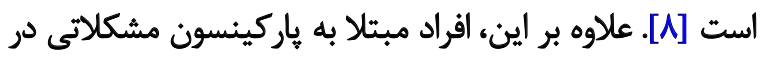

طبق تزارش سازمان جهانى بهداشت'، تعداد سالمندان جهان

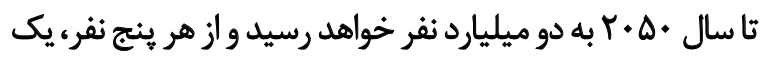

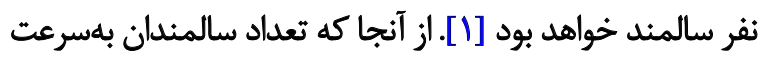

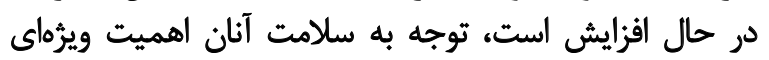

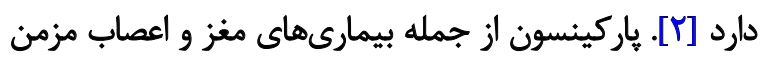

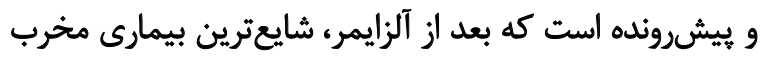

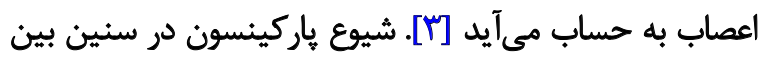

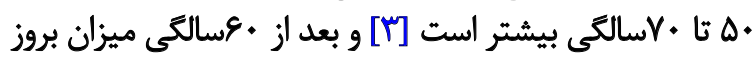

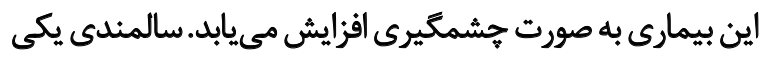
از برهداى خطرناك براى ابتلا به هاركينسون است؛ به به نحوى كه

1. World Health Organzation (WHO)

\section{$-$}


يرسشنامه و تمايلنداشتن به همكارى مراجعان در هر مرحله از

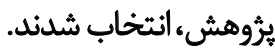

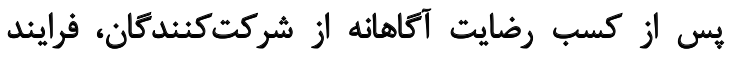

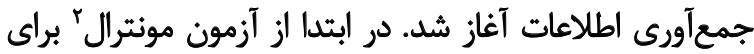

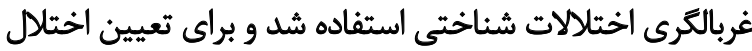

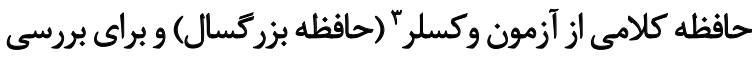

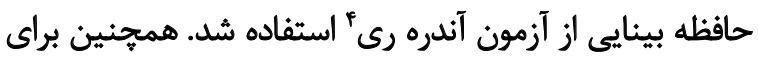

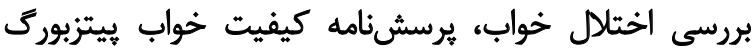

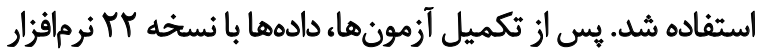

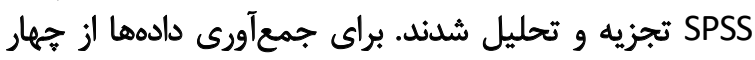

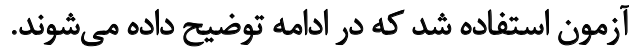

\section{أز مون شئاغتى موئترال}

آزمون شناختى مونترال يك ابزار ساده، معتبر و حساس براي افراي

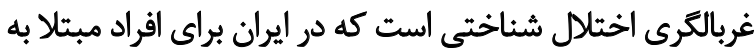

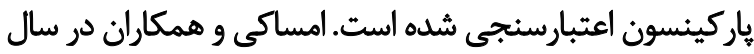

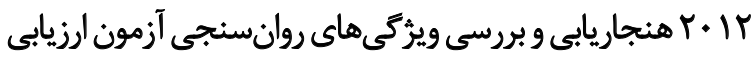

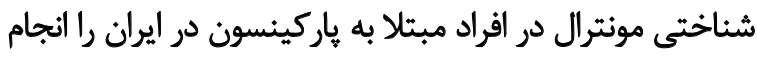

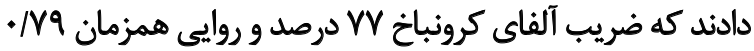

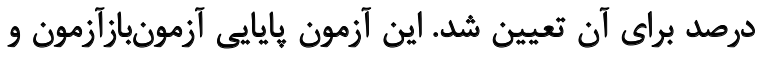

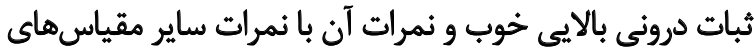

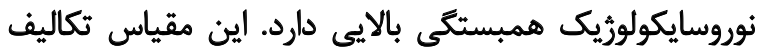

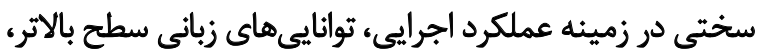

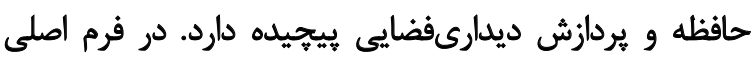

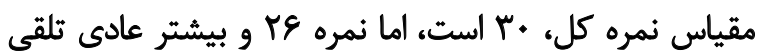

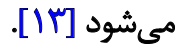

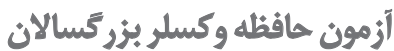

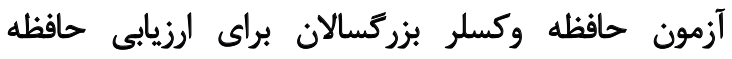

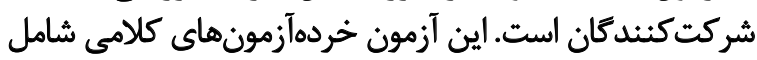

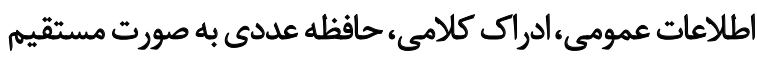

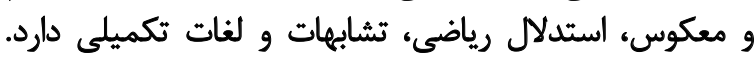

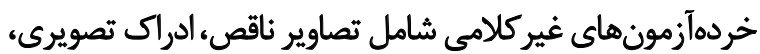
استدلال علمى، ساختن تصاوير و دقت و تداعى است اميد ساعدو همكاران اين آزمون رادر سال 9 × . باعتبارسنجى

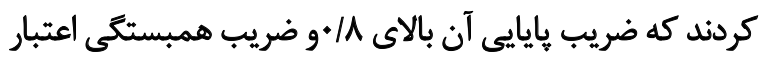

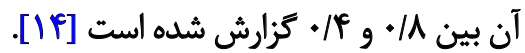

2. Montreal test

3. Wechsler

4. Andre Rey
زمينه عملكرد اجرايى، توجه، روابط فضايى و حافظه نيز دارند

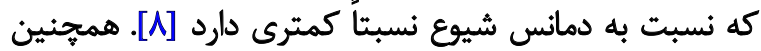

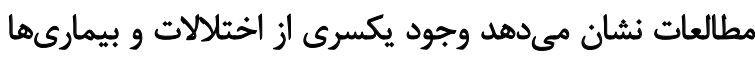

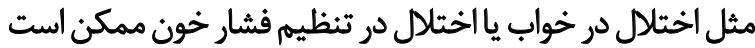

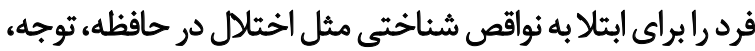

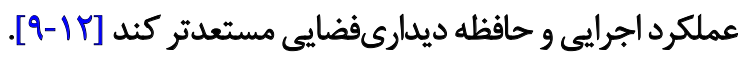

در مطالعهاى كه براي بررسى ارتباط بين اختلال خواب و ابتلا

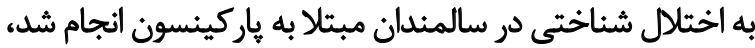

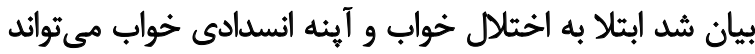

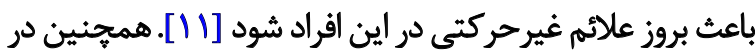

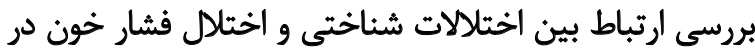

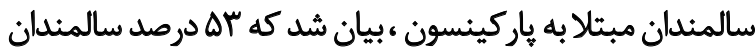

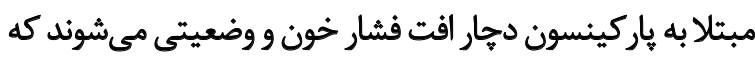

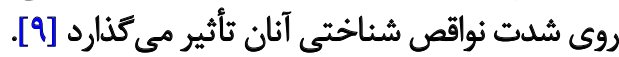
با توجه به مطالب بيانشده مى توان كفت برخى شاخصهاى

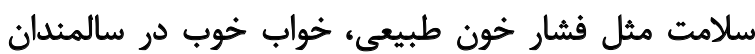

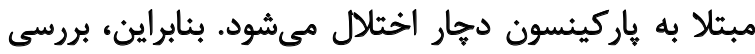

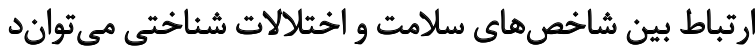

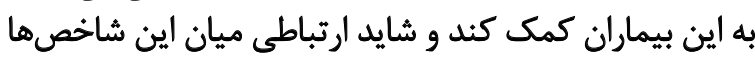

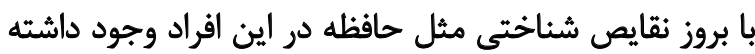

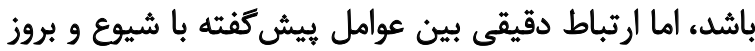

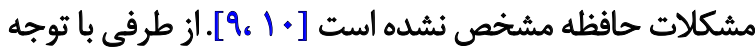

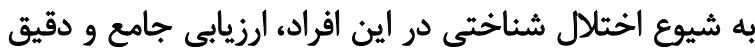

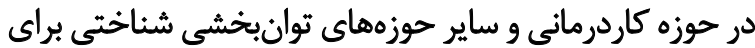

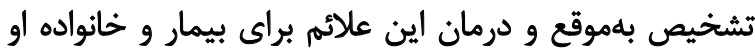

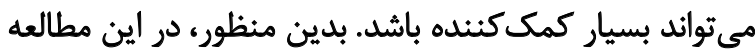

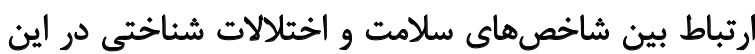
بيماران بررسى شد.

$$
\text { روش مطالعه }
$$

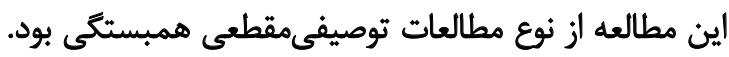

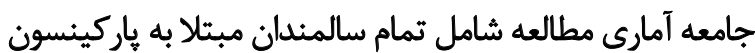

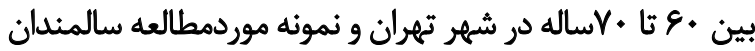

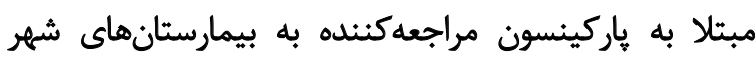

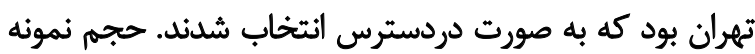

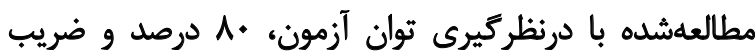

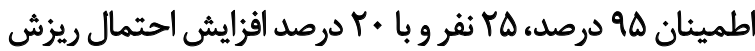

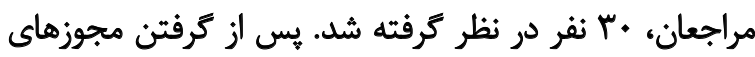

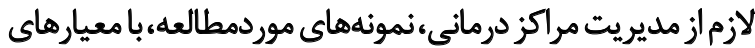

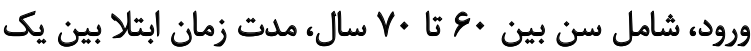

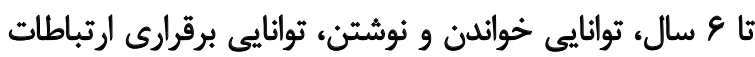

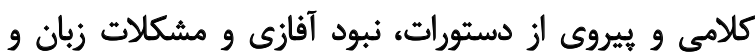
معيار هاى خروج شامل نبود روند صحيح ياسخغويى به سؤالات و نئون 


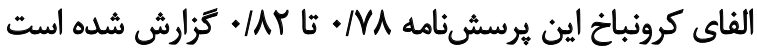

[19, IV]

يافتهها

نتايج بهدست آمده از بررسى • ب نمونه شركت كننده در اين

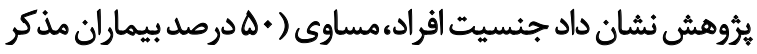

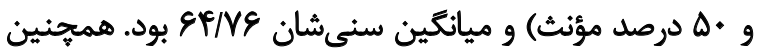

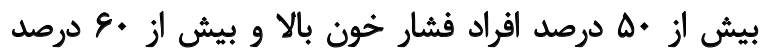

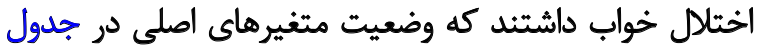

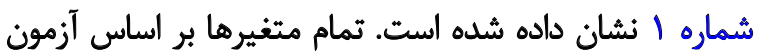

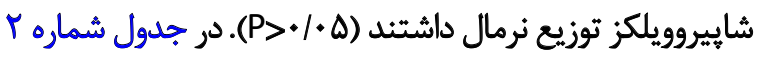

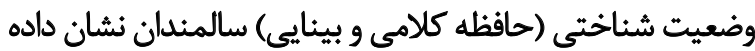

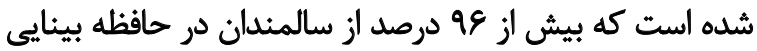

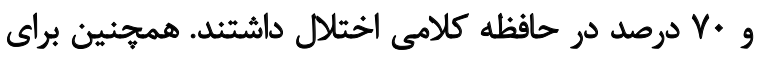

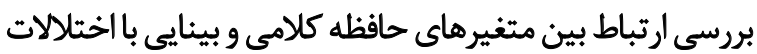

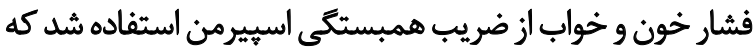

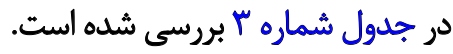

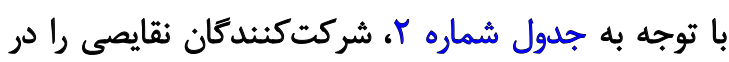

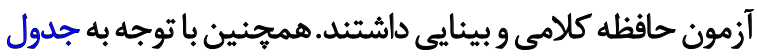

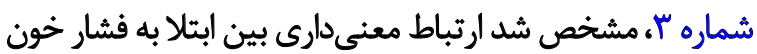

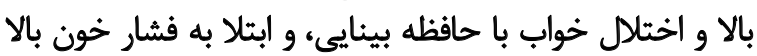

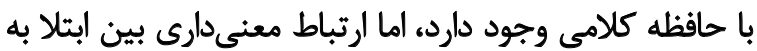
اختلال خواب و حافظه كلامى يبدا نشد.

بحث

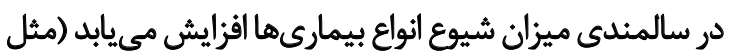

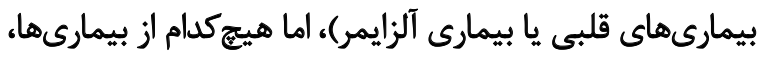

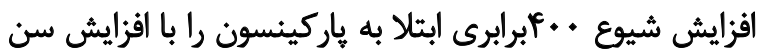

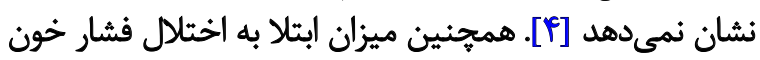

آزمون آندره رى براى بررسى ادراك و حافظه بينايى است،

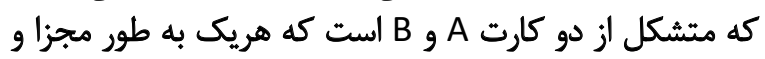

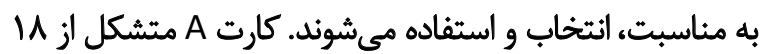

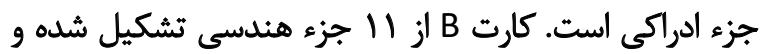

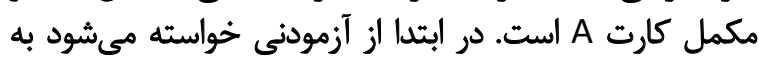

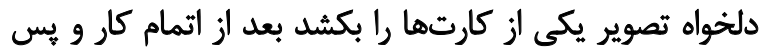

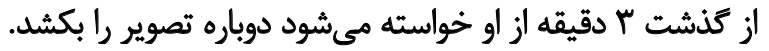

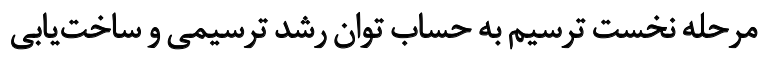

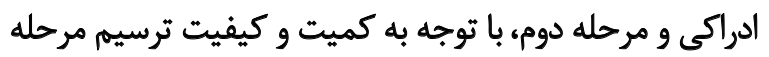

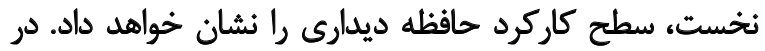

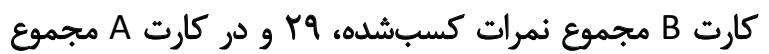

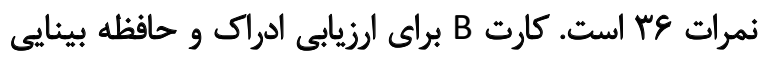

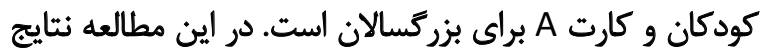

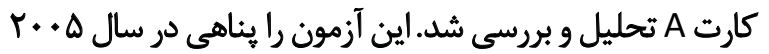

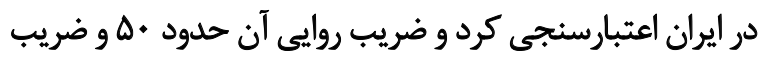

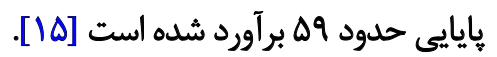

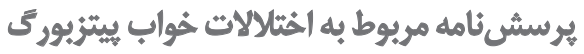

اين يرسشنامه براى تعيين كيفيت خواب است كه در اصل

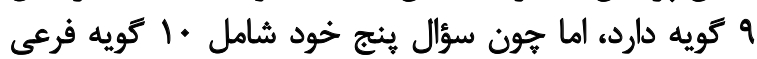

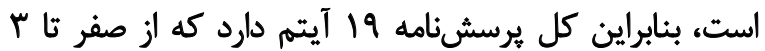

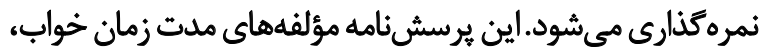

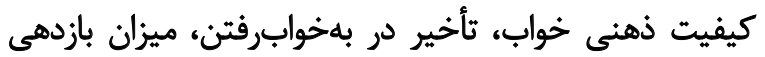

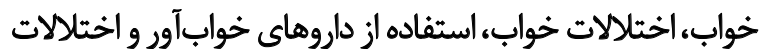

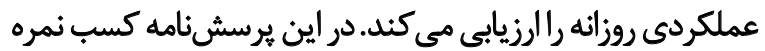

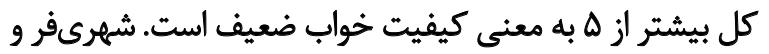

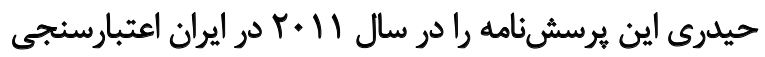

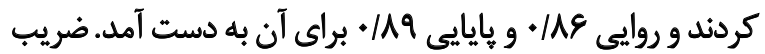

جدول 1. مشخصات جمعيتشناختى و شاخصهاى سلامت سالمندان مبثلا به ياركينسون (·r=r)

\begin{tabular}{|c|c|c|c|}
\hline درصد & تعداد & رده متغير & 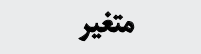 \\
\hline 0.1 & is & 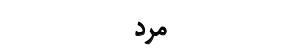 & \multirow[b]{2}{*}{ 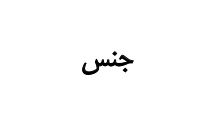 } \\
\hline$\Delta . /$ & 10 & زن & \\
\hline $\operatorname{rep}$ & if & اختلاللنداشتن در فشار خون & \multirow[b]{2}{*}{ اختلال فشار خُن } \\
\hline$\Delta \Psi / T$ & 18 & "اختلالداشتن در فشار خون & \\
\hline $\operatorname{sedV}$ & r. & اختلالداشتن در خواب & \multirow{2}{*}{ اختلال خواب } \\
\hline$\pi / \pi$ & 1. & اختّلال نداشتن خُواب & \\
\hline$E \leftarrow / V E \pm V / A V(\varepsilon \cdot \cdot V \Delta)$ & \multicolumn{2}{|c|}{ (ييشينه، كمينه) انحرافمعياربميانكين } & سن (بر حسب سال) \\
\hline
\end{tabular}


جدول r. توزيع فراوانى شاخصهاي شئاختى سالمئدان مبتلا به ياركييسون (•rn)

\begin{tabular}{|c|c|c|c|c|c|}
\hline انحراف معيار & ميانكين & حداكثر & حداقل & ره متغير & مثغير \\
\hline$\Delta / T^{N}$ & $r / q$ & me & + & & حاقظه بينائى )ى \\
\hline$|8| \cdot \Delta$ & $9+18$. & irq & 98 & نمره كل & \multirow{8}{*}{ وكسلر } \\
\hline $1 / 11$ & $e / M^{\prime}$ & 8 & $r$ & اطالاعات شخصىى & \\
\hline I/TA & T/VE & $\Delta$ & 1 & جهتيائى & \\
\hline r/If & f/qr & $\wedge$ & - & كثترل ذهنى & \\
\hline rpeq & $P / T$ & 1. & - & حافظه منطقى & \\
\hline $1 / 199$ & $N \cdot F$ & ir & $r$ & تكرار ارقام & \\
\hline P/TA & gl.r & if & - & حافظه بينايى & \\
\hline$r / q$. & II/Ar & 19 & $8 / 0$. & يادكيرى تداعىها & \\
\hline
\end{tabular}

جدول r. ضريب همبستكى بين حافظه كلامى و بينايع با شاخص هاى سلامت سالمثدان مبتلا به باركينسون (+rn)

\begin{tabular}{|c|c|c|c|}
\hline اختلال خواب & فشار خون & \multicolumn{2}{|c|}{ مثيخير } \\
\hline .1 .99 & $.10 . t$ & مقدار احتمال & \\
\hline$\cdot / \mu+V$ & $-+/ \Delta 1 f$ & ضريب همبستخى & \\
\hline .1 .40 & 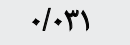 & مقدار احتمال & \\
\hline . MEA & $-A \mid$ ATI & ضريب همبستكي & حافظه بينايى \\
\hline
\end{tabular}

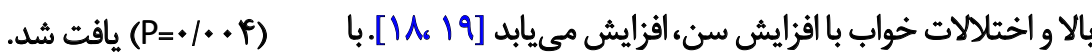

يس از جستوجوى يايگماههاى دادهها، يثوهش زيادى درباره

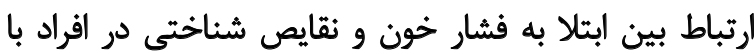

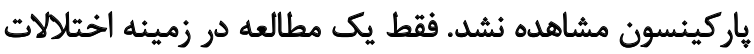
فشار خون و نقايص شناختى يافت شد كه نتايج اين مطالعه باليا

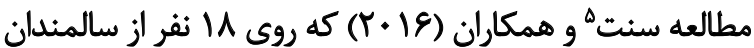

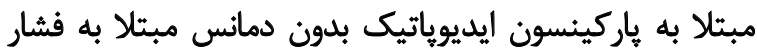

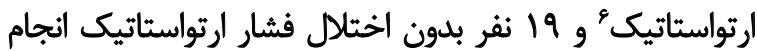

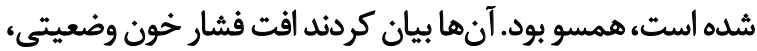

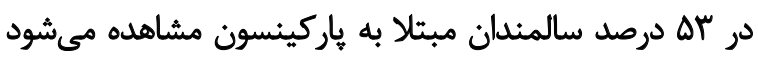

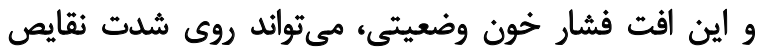

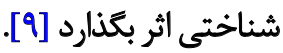

با توجه به مطالعه سيرالو همكاران كه به بررسي آسيب رك هاي

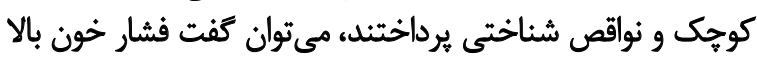

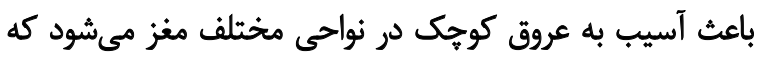

\footnotetext{
5. Centi

6. Orthostatic hypotension

7. Sierra
}

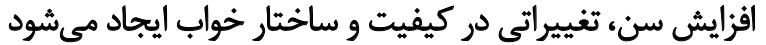

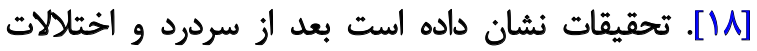
كوارشى، خواب باكيفيت ضعيف در رتبه سوم مشكلات سالمندان

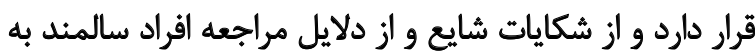

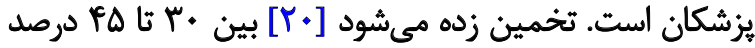

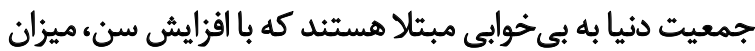

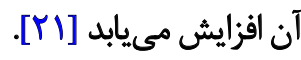
در اين يثوهش نيز بيش از • ه درصد از شركت كنيندها در

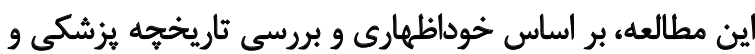

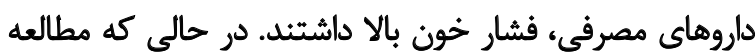

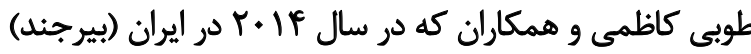

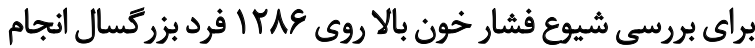

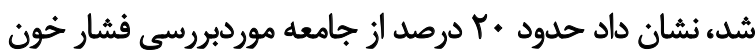

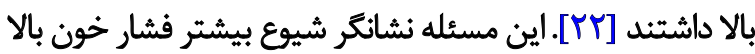

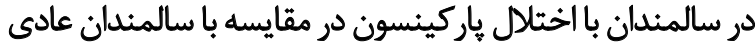

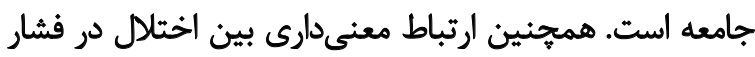

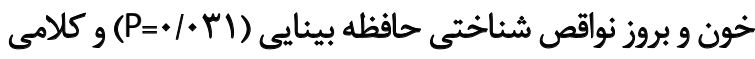


تأثير مستقيه بر عملكرد حافظه دارد و افرادى كه كيفيت خواب

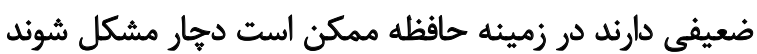

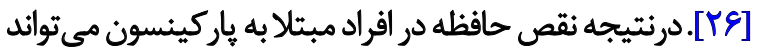
با مشكلات خواب ارتباط مستقيم داشته باشد.

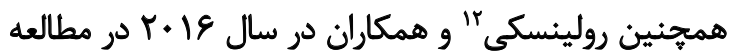

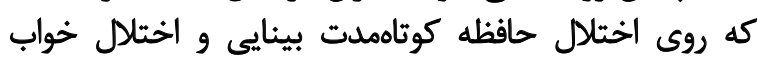

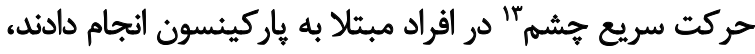

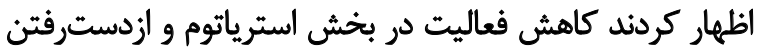

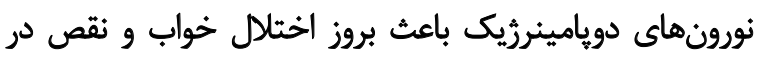
حافظه كوتامدت بينايى مي شوينود [YrV]

نتايج در اين مطالعه نشان داد، بين اختلال خواب و اختاللال

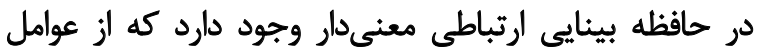

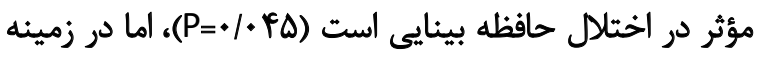

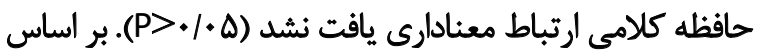

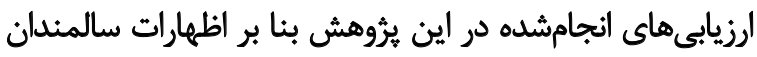

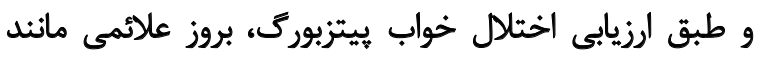

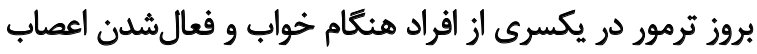

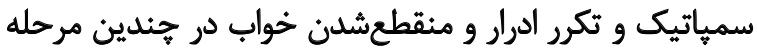

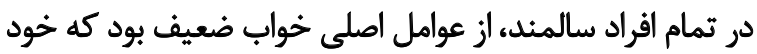

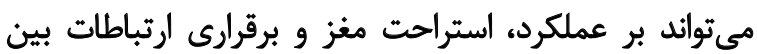

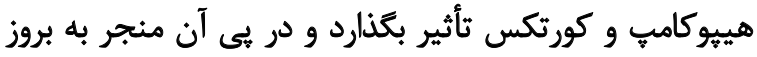

نواقص شناختى شود [عrك].

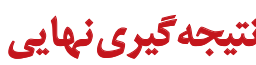

نتايج اين مطالعه نشان داد ارتباط معنى دارى بين شاخصهاي

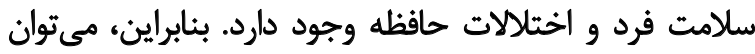

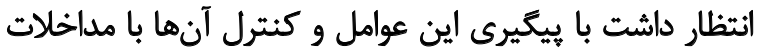

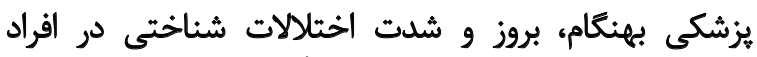

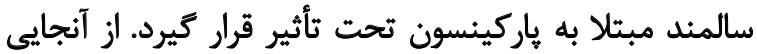

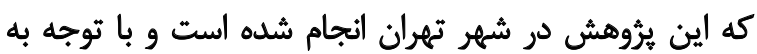

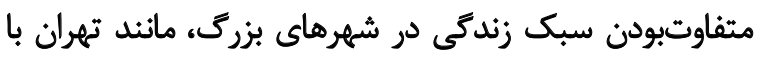

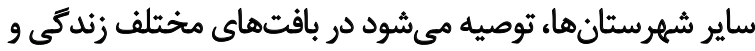

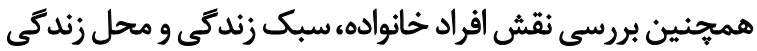

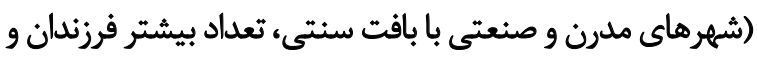

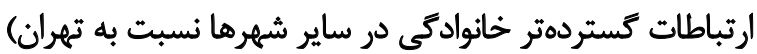
يرؤرشهايى انجام شود.

براى ارزيابى بهتر از وضعيت حافظه افراد سالمئد با ياركينسون،

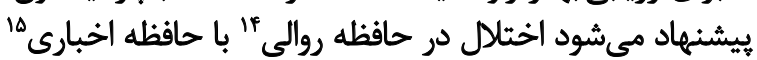
مقايسه شُود.

12. Rolinski

13. Rapid eye movement

14. Non-Declerative

15. Declarative
عارضه قابلمشاهداهي مثل سكته مغزى در افراد ايجاد نمى كند، اما

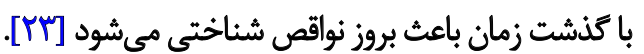

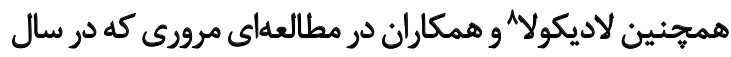

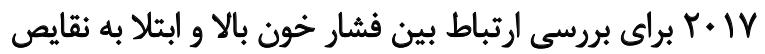

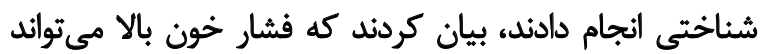

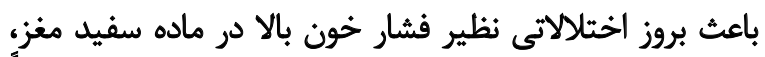

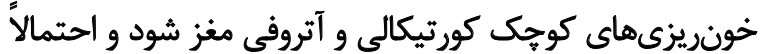

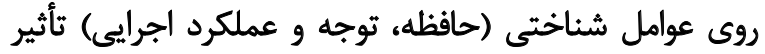

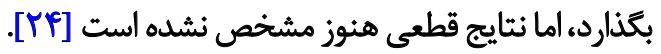

با توجه به ماهيت بيمارى هاركينسون در ايجاد اختلال بين

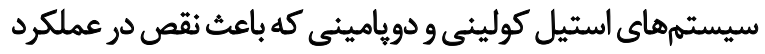

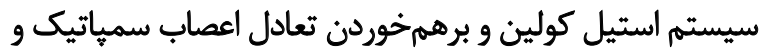

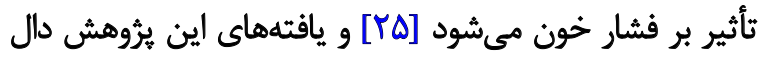

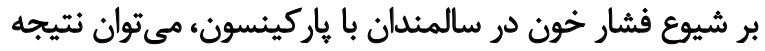

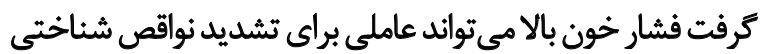
در مبتلايان به باركينسون شود.

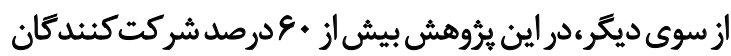

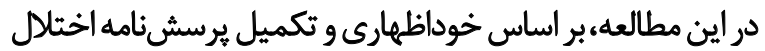

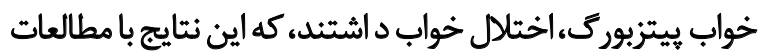

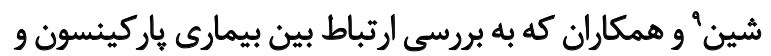

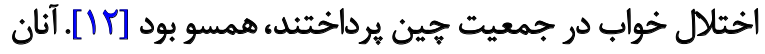

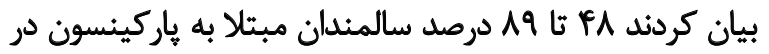

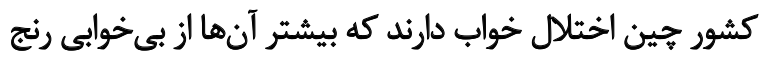

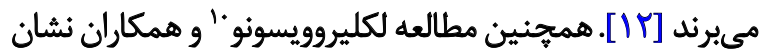

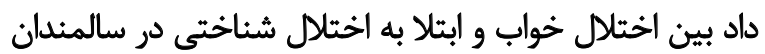

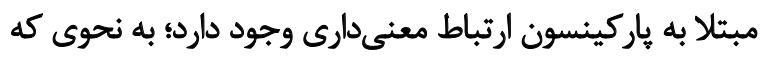

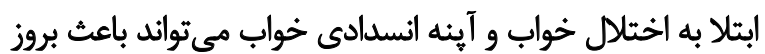

علائم غير حركتى در اين افراد شود [11]

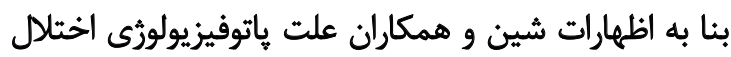

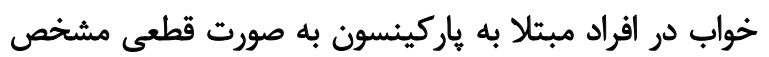

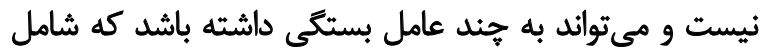

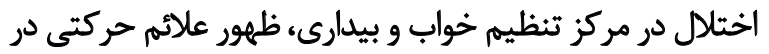

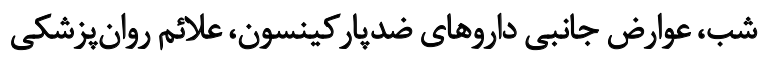

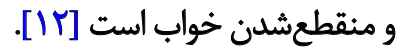

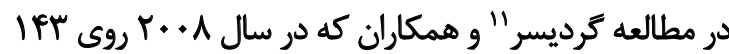

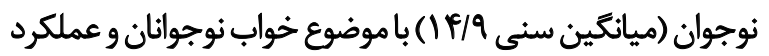

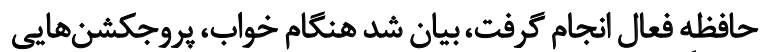

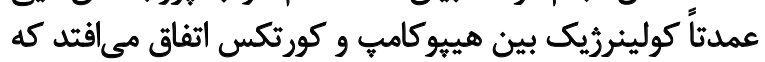
8. Ladecola
9. Shen
10. Leclair-Visonneau
11. Gradisar 


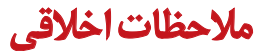

\section{ييروى أز اصول اخلاق يثوهش}

اين مقاله از دانشكاه علوم بهزيستى و توانبخشى كد اخلاقى

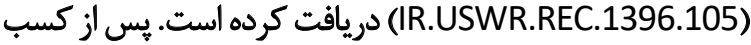

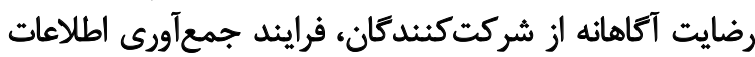

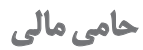

مقاله بركرفته از باياننامه سيده مطهره حجازى در كروه

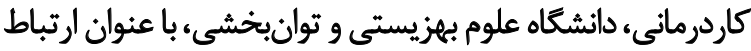

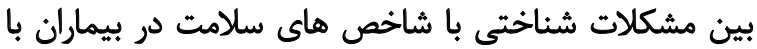

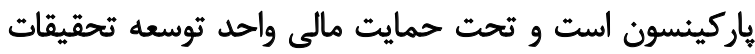
بالينى بيمارستان توانبخشى رفيده بون بوده است

$$
\text { مشاركت نويسند متان }
$$

مفهوم سازى: سيده مطهره حجازي، نازيلااكبر فهيمي، حجت

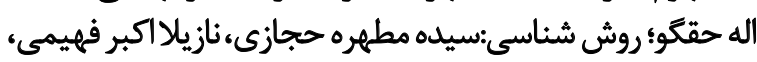

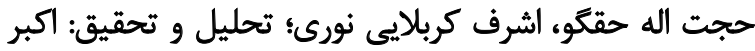

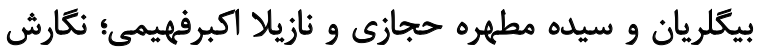

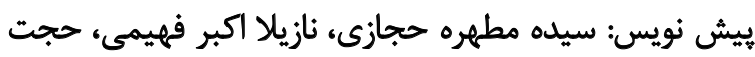

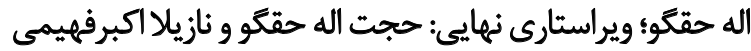

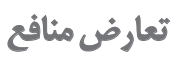

بنا به اظهار نويسندكان، در اين مقاله هيجّمونه تعارض منافعى

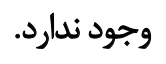

$$
\text { تشكر و قدردافئ }
$$

از تمامى سالمندان ترامى شركتكنتنده در اين مطالعه،

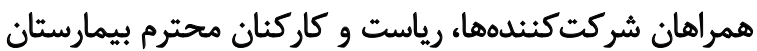

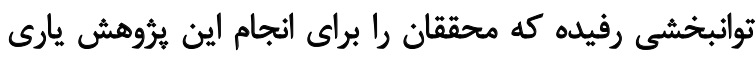

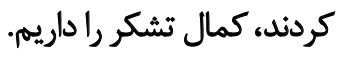




\section{References}

[1] World Health Organization. World Health Statistics 2012. Geneva: World Health Organization; 2012.

[2] Habibi A, Nikpour S, Seyedoshohadaei M, Haghani H. [Health promoting behaviors and its related factors in elderly (Persian)] Iran Journal of Nursing. 2006; 19(47):35-48.

[3] Kalia LV, Kalia SK, Lang AE. Disease-modifying strategies for Parkinson's disease. Movement Disorders. 2015; 30(11):1442-50. [DOI:10.1002/mds.26354] [PMID]

[4] Rodriguez M, Rodriguez Sabate C, Morales I, Sanchez A, Sabate M. Parkinson's disease as a result of aging. Aging Cell. 2015; 14(3):293-308. [DOI:10.1111/acel.12312] [PMID] [PMCID]

[5] Goetz CG. The history of Parkinson's disease: Early clinical descriptions and neurological therapies. Cold Spring Harbor Perspectives in Medicine. 2011; 1(1):a008862. [DOI:10.1101/cshperspect.a008862] [PMID] [PMCID]

[6] Litvan I, Aarsland D, Adler CH, Goldman JG, Kulisevsky J, Mollenhauer B, et al. MDS task force on mild cognitive impairment in Parkinson's disease: Critical review of PD_MCI. Movement Disorders. 2011; 26(10):1814-24. [DOI:10.1002/mds.23823] [PMID] [PMCID]

[7] Azuma T, Cruz RF, Bayles KA, Tomoeda CK, Montgomery EB. A longitudinal study of neuropsychological change in individuals with Parkinson's disease. International Journal of Geriatric Psychiatry. 2003; 18(12):1115-20. [DOI:10.1002/gps.1022] [PMID]

[8] Petersen RC. Mild cognitive impairment as a diagnostic entity. Journal of Internal Medicine. 2004; 256(3):183-94. [DOI:10.1111/ j.1365-2796.2004.01388.x] [PMID]

[9] Centi J, Freeman R, Gibbons CH, Neargarder S, Canova AO, Cronin Golomb A. Effects of orthostatic hypotension on cognition in Parkinson disease. Neurology. 2017; 88(1):17-24. [DOI:10.1212/ WNL.0000000000003452]

[10] Chahine LM, Weintraub D, Hawkins KA, Siderowf A, Eberly $\mathrm{S}$, Oakes D, et al. Cognition in individuals at risk for Parkinson's: Parkinson Associated Risk Syndrome (PARS) study findings. Movement Disorders. 2016; 31(1):86-94. [DOI:10.1002/mds.26373] [PMID] [PMCID]

[11] Leclair Visonneau L, Clairembault T, Coron E, Le Dily S, Vavasseur F, Dalichampt M, et al. REM sleep behavior disorder is related to enteric neuropathology in Parkinson disease. Neurology. 2017; 89(15):1612-8. [DOI:10.1212/WNL.0000000000004496] [PMID]

[12] Shen Y, Liu CF. Sleep disorders in Parkinson's disease: Present status and future prospects. Chinese Medical Journal. 2018; 131(8):883-5. [DOI:10.4103/0366-6999.229903] [PMID] [PMCID]

[13] Emsaki G, Molavi H, Chitsaz A, Abtahi MM, Asgari K. [Psychometric properties of the Montreal Cognitive Assessment (MoCA) in Parkinson's disease patients in Isfahan (Persian)]. Journal of Isfahan Medical School. 2011; 29(158):1391-400.

[14] Saed O, Rushan R, Moradi A. [Investigating psychometric properties of Wechsler Memory Scale-for the students of Tehran Universities (Persian)]. Clinical Psychology \& Personality. 2008; 1(31):57-70.

[15] Nazaribadie M, Asgari K, Amini M, Abedi A. [An investigation of the cognitive performances in patients with type 2 diabetes in comparison to pre-diabetic patients (Persian)]. Advances in Cognitive Science. 2011; 13(3):33-40.

[16] Buysse DJ, Reynolds CF, Monk TH, Berman SR, Kupfer DJ. The Pittsburgh Sleep Quality Index: A new instrument for psychiatric practice and research. Psychiatry Research. 1989; 28(2):193-213. [DOI:10.1016/0165-1781(89)90047-4]

[17] Heidari A, Ehteshamzadeh P, Marashi M. [The relationship between insomnia intensity, sleep quality, sleepiness and mental health disorder with educational performance in female adolescences of Ahwaz city (Persian)]. Woman and Culture. 2010; 1(4):65-76.

[18] Reid KJ, Martinovich Z, Finkel S, Statsinger J, Golden R, Harter $\mathrm{K}$, et al. Sleep: A marker of physical and mental health in the elderly. The American Journal of Geriatric Psychiatry. 2006; 14(10):860-6. [DOI:10.1097/01.JGP.0000206164.56404.ba] [PMID]

[19] Ahangari M, Kamali M, Arjmand hesabi M. [The effects of high blood pressure on the quality of life of the elderly in the elderly cultural center of Tehran (Persian)]. Iranian Journal of Aging. 2008; 3(7):26-32

[20] Cotroneo A, Gareri P, Lacava R, Cabodi S. Use of zolpidem in over 75-year-old patients with sleep disorders and comorbidities. Archives of Gerontology and Geriatrics Supplement. 2004; (9):936. [DOI:10.1016/j.archger.2004.04.015] [PMID]

[21] Leger D, Poursain B, Neubauer D, Uchiyama M. An international survey of sleeping problems in the general population. Current Medical Research and Opinion. 2008; 24(1):307-17. [DOI:10.1185/030079907X253771] [PMID]

[22] Kazemi T, Hajihosseini M, Mashreghimoghadam H, Azdak $\mathrm{N}$, Ziaee M. Prevalence and determinants of hypertension among Iranian adults, Birjand, Iran. International Journal of Preventive Medicine. 2017; 8:36.

[23] Sierra C. Cerebral small vessel disease, cognitive impairment and vascular dementia. Panminerva Medica. 2012; 54(3):179-88 [PMID]

[24] Iadecola C, Yaffe K, Biller J, Bratzke LC, Faraci FM, Gorelick $\mathrm{PB}$, et al. Impact of hypertension on cognitive function: A scientific statement from the American Heart Association. Hypertension. 2016; 68(6):e67-e94. [DOI:10.1161/HYP.0000000000000053] [PMID] [PMCID]

[25] Rizzi G. Dopamine and acetylcholine, a circuit point of view in Parkinson's disease. Frontiers in Neural Circuits. 2017; 11:110. [DOI:10.3389/fncir.2017.00110] [PMID] [PMCID]

[26] Gradisar M, Terrill G, Johnston A, Douglas P. Adolescent sleep and working memory performance. Sleep and Biological Rhythms. 2008; 6(3):146-54. [DOI:10.1111/j.1479-8425.2008.00353.x]

[27] Rolinski M, Zokaei N, Baig F, Giehl K, Quinnell T, Zaiwalla $Z$, et al. Visual short-term memory deficits in REM sleep behaviour disorder mirror those in Parkinson's disease. Brain. 2015; 139(1):47-53. [DOI:10.1093/brain/awv334] [PMID] [PMCID] 